\title{
The Effect of Injected Energy on Low Energy Single Longitudinal Mode Pre-Lase Q-Switched Pr:YLF Laser
}

\author{
Weicheng Dai, Long Jin, Yuan Dong, Xihe Zhang, Guangyong Jin* \\ The Key Laboratory of Jilin Province Solid-State Laser Technology and Application, Changchun University of Science and \\ Technology, Changchun, China \\ Email: *jgycust@163.com
}

How to cite this paper: Dai, W.C., Jin, L., Dong, Y., Zhang, X.H. and Jin, G.Y. (2019) The Effect of Injected Energy on Low Energy Single Longitudinal Mode Pre-Lase Q-Switched Pr:YLF Laser. Journal of Applied Mathematics and Physics, 7, 29682978.

https://doi.org/10.4236/jamp.2019.712207

Received: November 20, 2019

Accepted: December 2, 2019

Published: December 9, 2019

\begin{abstract}
In this paper, the effect of injected energy on low energy single longitudinal mode (SLM) pre-lase Q-switched is analyzed and the optimization procedure is shown in detail. Here, taking the Pr:YLF laser as an example of low energy laser, and the parameters of Pr:YLF laser by using pre-lase have been shown. Compared with normal Q-switched laser, the single pulse energy reaches $60.16 \%$ and the pulse width exceeds $39.73 \%$ when the same maximum energy is injected and SLM is achieved in pre-lase. The analysis results show that pre-lase is suitable for low energy laser to obtain SLM and there be an optimal performance to achieve the optimal energy output.
\end{abstract}

\section{Keywords}

Pre-Lase Q-Switched, Single Longitudinal Mode, Injected Energy, Pr:YLF

\section{Introduction}

The efficiency and energy of SLM is a primary problem which has limited the application of Q-switched laser in many fields for a long time. Especially when a low energy laser is tried to modulate to be SLM, the output energy often couldn't be measured and used. In fact, people often gets SLM Q-switched laser by three methods: 1) Introducing some devices to increase the loss among different modes and keeping central mode have the lowest loss [1] [2] [3] [4] [5], the central mode would survive and other modes would disappear after the initial oscillation. The device which controls the loss of different modes can be designed and applied simply, and it is usually used in high energy laser to get SLM because the loss of the device is too high to get the output energy remain a lot. 2) 
Extending the stage at which the different modes begin to compete [6] [7] [8], then central mode would get the maximum gain than the others, because the single trip gain of central mode is higher than other modes. The time of extending stage is limited by the gain identification. It is not fit for some high energy laser to get SLM, because the gain identification is effected greatly by injected energy. 3) Creating a high quality laser as a seed laser and coupling the seed laser into the another laser instead of initial noise [9] [10] [11], then the SLM laser also could be obtained. The defect is that two lasers are needed at least and the design of coupling device is required strictly which tremendously limits the practical application. In fact, people often gets SLM by combining multifarious ways when the injected energy is enough high [12], and pre-lase is an effective way to get SLM laser if the energy of original laser is limited by the existing technology.

Pre-lase is a combination technique of the method (2) and (3) by which the SLM laser can be obtained. After the pump energy is injected, the procedure of pre-lase can be described as below: First, the loss of Q-switched is reduced to a low value instantly and the seed signal is produced, then the seed signal begin to oscillate and different modes begin to compete in the steady-state in which the mode competition starts. Finally, the central mode survives and other modes disappear after the oscillation. Switch the loss to be zero, and then the SLM pulse will come into being. Pr:YLF laser is a new type laser and the abundant wavelengths $(479 \mathrm{~nm}, 522 \mathrm{~nm}, 607 \mathrm{~nm}, 639 \mathrm{~nm}, 747 \mathrm{~nm}$ can be acquired directly, and $304 \mathrm{~nm}, 374 \mathrm{~nm}$ can be efficiently obtained after the double-frequency) attract many scientists to pay attention [13] [14] [15] [16], but the application of Pr:YLF laser is limited by the low energy. So, it is necessary to do the research on how to make the Pr:YLF laser turn into SLM. In this paper, the optimized parameters and the best output characters are shown when the injected energy is changed and the maximum energy is reached. After the calculation and analysis, the result shows that the output characters of pre-lase Q-switched can be close to the normal Q-switched and superior to the other ways of obtaining SLM when the energy of laser is limited by the existing technology.

\section{The Model of Gain Identification}

In fact, pre-lase is a technique in which the seed signal (The quality of seed signal is not as good as the pure seed laser, but it is much better than the noise) can be produced by itself and central mode can be amplified to a maximum value after the oscillation. In the whole pre-lase process, there are three parts need to be considered: the gain identification, the optimization of mode competition and the output process.

Here the model of gain identification is established and analyzed. Firstly, the threshold inversion population and threshold value can be obtained from the four-level rate equation [17]:

$$
\Delta n_{t}=\frac{L}{2 \sigma l}
$$




$$
E_{t}=\frac{h v \Delta n_{t} \pi r^{2} l}{\eta_{0}}
$$

where $L=0.04$ is the optical loss of round trip, $\sigma=1.4 \times 10^{-19} \mathrm{~cm}^{2}$ is the cross-section of the central mode $(607 \mathrm{~nm})$ in the Pr:YLF laser, $l=9 \mathrm{~mm}$ is the medium length, $v=6.76 \times 10^{14} \mathrm{HZ}$ is the frequency of central mode, $r=300 \mu \mathrm{m}$ is the radius of oscillating beam cross-section, $\eta_{0}=1$ is the efficiency of the pump, $h=6.62617 \times 10^{-34} \mathrm{~J} \cdot \mathrm{s}$.

Set the Q-switched loss and steady-state loss $L_{x}=X L, L_{k}=K L$. The inversion population at and after the Q-switched can be given respectively: $n_{x}=X \Delta n_{t}, n_{k}=K \Delta n_{t}$. While the loss changes slightly and the seed signal produces, the inversion population consumed by seed signal can be described as $\Delta n_{0}=(X-K) \Delta n_{t} .(X-K<1$, if $X-K>1$, the laser would be produced and the seed signal would disappear.)

When the loss is switched to $L_{x}$, the full width at half maximum (FWHM) of the seed signal oscillation can be given:

$$
\frac{n_{0}}{\left(\frac{\Delta v_{s}}{2}\right)^{2}+\left(\frac{\Delta v_{D}}{2}\right)^{2}}=\frac{\Delta n_{t}}{\left(\frac{\Delta v_{D}}{2}\right)^{2}}
$$

Settling the Equation (3) and the simplified result is shown in Equation (4):

$$
\Delta v_{s}=\sqrt{X-1} \Delta v_{D}
$$

The spacing of two adjacent longitudinal modes $\Delta v$ which is decided by the specified length resonant cavity also can be obtained:

$$
\Delta v=\frac{c}{2\left[l \cdot n+\left(l_{0}-l\right)\right]}
$$

where $\Delta v_{D}=100 G H Z$ is the FWHM of Pr:YLF spontaneous radiation, $\Delta v_{s}$ is the FWHM of the seed signal oscillation when the system is at the state of Q-switched, the number of longitudinal modes can be described as $N=\frac{\Delta v_{s}}{\Delta v}$ ( $N$ should be positive integer), $n=1.45$ is the refractive index of Pr:YLF crystal, $l_{0}=20 \mathrm{~mm}$ is the length of resonant cavity.

Pr:YLF laser is the solid laser which belongs to the homogeneous broadening, introduce the atomic linear function of homogeneous broadening:

$$
\tilde{g}(v)=\frac{g_{0}}{2 \pi}\left[\left(v-v_{0}\right)^{2}+\left(\frac{\Delta v_{s}}{2}\right)^{2}\right]^{-1}
$$

where $\tilde{g}(v)$ is the gain of different modes, $g_{0}$ is the gain of central mode which is decided by the characters of the laser. The center mode and the adjacent mode almost occupy the gain much more than others at the stage of the competition, so the gain of the other mode can be ignored. The ratio of the gain difference between adjacent mode $v_{1}$ (there are two symmetrical modes nearby the central mode, and take one as an example) and central mode $v_{0}$ which is the gain identification can be obtained from Equation (6): 


$$
\frac{g_{0}-g_{1}}{g_{0}}=\frac{\left(v_{1}-v_{0}\right)^{2}}{\left(v_{1}-v_{0}\right)^{2}+\left(\frac{\Delta v_{s}}{2}\right)^{2}}
$$

Substituting Equation (4) into Equation (7), we can get Equation (8) which shows the relation between the injected energy and the gain identification:

$$
\frac{g_{0}-g_{1}}{g_{0}}=\frac{1}{1+\frac{X-1}{4}\left(\frac{\Delta v_{D}}{\Delta v}\right)^{2}}
$$

According to Figure 1, it can be known that gain identification becomes smaller when the injected energy increases. The root cause is that the increased FWHM would arouse more longitudinal modes, and the distribution of energy among different modes becomes to be much uniform. It means that the central mode would have seriously encroached and the SLM would be failed. It is the main reason that the high energy laser is limited to get SLM, the gain difference cannot be modulated artificially and SLM requires the gain or loss between different modes high enough. Finally, some scientists found a simple way to get the SLM laser that introduces some device to make different modes own different losses, and it can be controlled easily. If the energy of the laser is high enough, SLM laser can be acquired simply. While the energy of the laser is low and the loss device is introduced, the SLM laser would be too weak to be detected [18]. So, it would be better to get the SLM without any loss device when the energy of the laser is low.

Pre-lase is a high-efficiency way of obtaining the SLM in low energy laser, but it is limited by the ratio of gain between central mode and adjacent mode. If the ratio of gain is high enough, the gain of a single trip will be higher and the number of the round trip will decrease which leads less time cost on the stage of

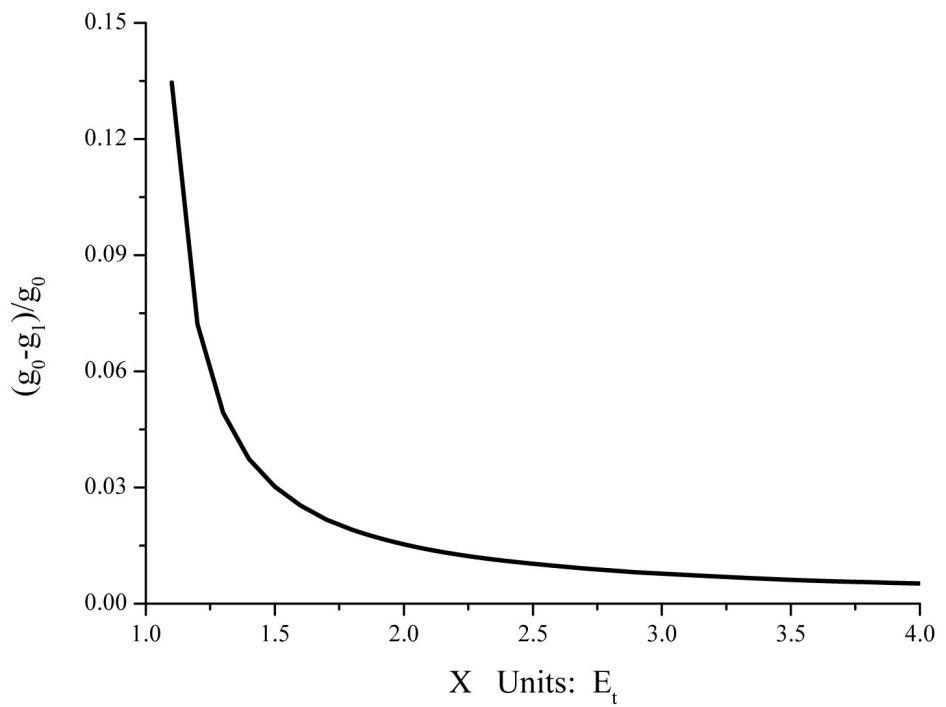

Figure 1. The relation between the ratio of the gain identification and the injected energy is shown. 
mode competition. In contrast, the time cost on the stage of mode competition will be more. While the time cost is longer than the lifetime of spontaneous emission, the spontaneous emission will get the leading and the energy of the central mode will decrease rapidly. The ratio of gain between central mode and adjacent mode decides the whole process of pre-lase, so to speak.

\section{The Model and Optimization of Competition}

When the seed signal starts to oscillate instead of the noise, there are two processes that have happened at the same time. The first process is the competition of the whole system in which the central mode, other modes and the loss of round trip consume the inversion population of the whole system collectively. The second process is the competition among different modes in which the central mode gets more energy than other modes and the SLM laser is achieved.

\subsection{The Model of the Whole Competition}

In the first process, the seed signal is amplified and the central mode consumes the most inversion population. Or more accurately, it is again optimization process in which the inversion population of central mode consumed by central mode should be equal to the inversion population offered by the whole system, finally, the central mode gets the maximum inversion population.

The inversion population of the whole system is distributed to three aspects, most are consumed on gaining the central mode, a part is consumed on the other modes and another part is used to overcome the loss of round trip. If the time cost on the competition of the whole system extends, the central mode will get the more inversion population and the stability of SLM will get better, but the loss of round trip will be higher. The time cost on the competition should be less than the spontaneous emission lifetime because the spontaneous emission influences the stability of the SLM seriously. Here the time cost on the competition is set as a limited value $t=35.7 \mu \mathrm{s}$ (which is the spontaneous emission lifetime of Pr:YLF.). The model of optimization will be established in order to ensure the competition carries on adequately.

Firstly, at the stage of Q-switched which is also the initial moment of the competition, the gain of single-trip can be described as follows [19]:

$$
G=\exp \left(2 \sigma_{n} n_{x} l\right)
$$

When the competition just finishes, the loss should be equal to the gain in the single-trip:

$$
L_{k}=G_{k}=\exp \left(2 \sigma_{n} n_{k} l\right)
$$

The inversion population consumption of the central mode is generated by the oscillation and amplification of the seed signal at the competition stage, and it can be obtained: 


$$
n_{1}=\frac{\Delta n_{0}}{N} \cdot\left[\left(\frac{G}{L_{k}}\right)^{\frac{1}{2}}\right]^{q}
$$

where $\left(\frac{G}{L_{k}}\right)^{\frac{1}{2}}=e^{\sigma_{n}(X-K) \Delta n l}$ is approximate to gain factor of a single trip, $\frac{\Delta n_{0}}{N}$ is the inversion population of central mode in the original seed signal, $q=\frac{c \cdot t}{2 l_{0}}$ is the number of circulation.

The inversion population which can be supplied to the center mode by the whole system is given:

$$
n_{2}=n_{k} \cdot\left(\frac{p_{0}}{p_{0}+2 p_{1}}\right) \cdot\left(\frac{1}{1+\left(\frac{L_{k}}{G}\right)^{\frac{q}{2}}}\right)
$$

In Equation (10), $n_{k}$ is the total gain inversion population provided by the system, $p_{0}, p_{1}$ are the power of central mode and adjacent mode, $\frac{p_{0}}{p_{1}} \geq 10$ is the precondition of SLM, suppose $\frac{p_{0}}{p_{1}}=10$ and the SLM is just achieved, $\frac{p_{0}}{p_{0}+2 p_{1}}=\frac{5}{6}$ is the factor of mode discrimination, $\left(\frac{L_{k}}{G}\right)^{\frac{1}{2}}$ is approximate to loss factor of single-trip. There is the relation between $n_{1}$ and $n_{2}$ in the process of oscillation, the efficiency of effective gain $\eta=\frac{n_{1}}{n_{2}}$ can be obtained as the Equation (11) shown:

$$
\eta=\frac{6(X-K)}{5 N \cdot K}\left[\exp \left(\sigma_{n} \Delta n c t(X-K) \frac{l}{l_{0}}\right)+1\right]
$$

Here, take $X=2$ as an example. After the oscillation, the inversion population of central mode and offered inversion population by the whole system can be shown in Figure 2.

The optimization process can be clearly acquired from Figure 2, the inversion population consumed by the central mode and the inversion population offered by the whole system decline when the loss of competition increases gradually. When the loss of competition gets to be a specific value which is shown in Figure 2, the value of $n_{1}$ gets to be equal to $n_{2}$ and the specific value is the extreme value. The population inversion consumed by the central mode will be more than the population inversion offered by the whole system if the extreme value is not reached, and the actual gain is the population inversion consumed by the central mode. If the extreme value is exceeded, the inversion population consumed by the central mode will be less than the inversion population offered 
by the whole system and the actual gain is the inversion population offered by the whole system. It is easy to see that the best optimization of central mode will be realized when the value of effective gain $\eta$ gets to be 1 .

After the above process, the relation among the injected energy, actual gain of central mode and loss set at the stage of competition can be obtained by the iterative computations which are shown in Figure 3.

From Figure 3, it can be seen that the actual gain of central mode could be changed nearly-linear when the injected energy increases and the loss at the stage of competition gets to be adjusted nearly-linear. It can be found the curve of the actual gain of central mode and the loss at the stage of the competition are not parallel and the slope of the loss at the stage of the competition is higher than the actual gain of central mode.

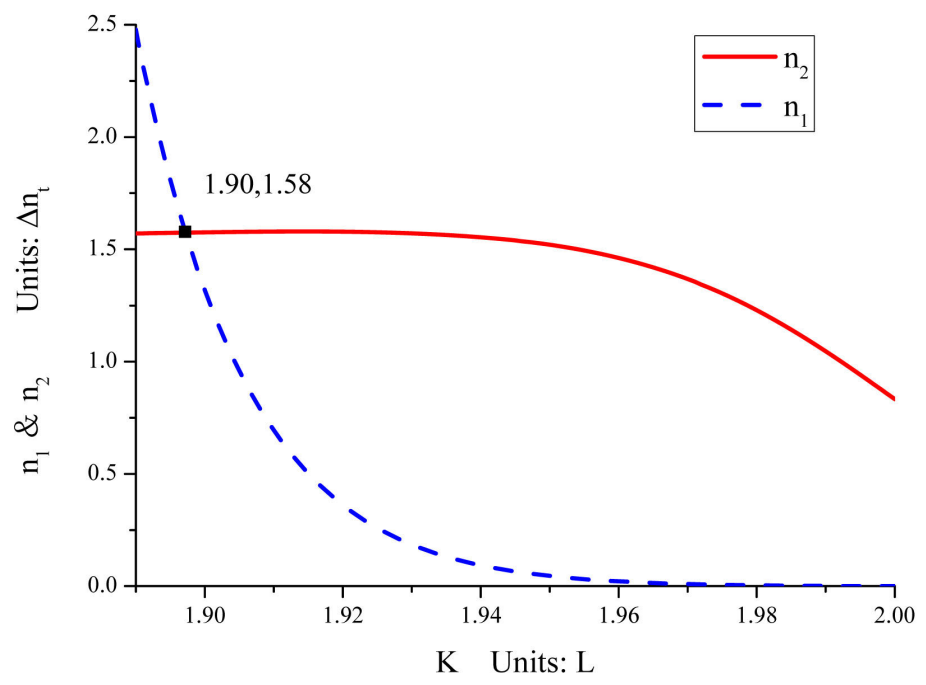

Figure 2. The optimization of the central mode is shown.

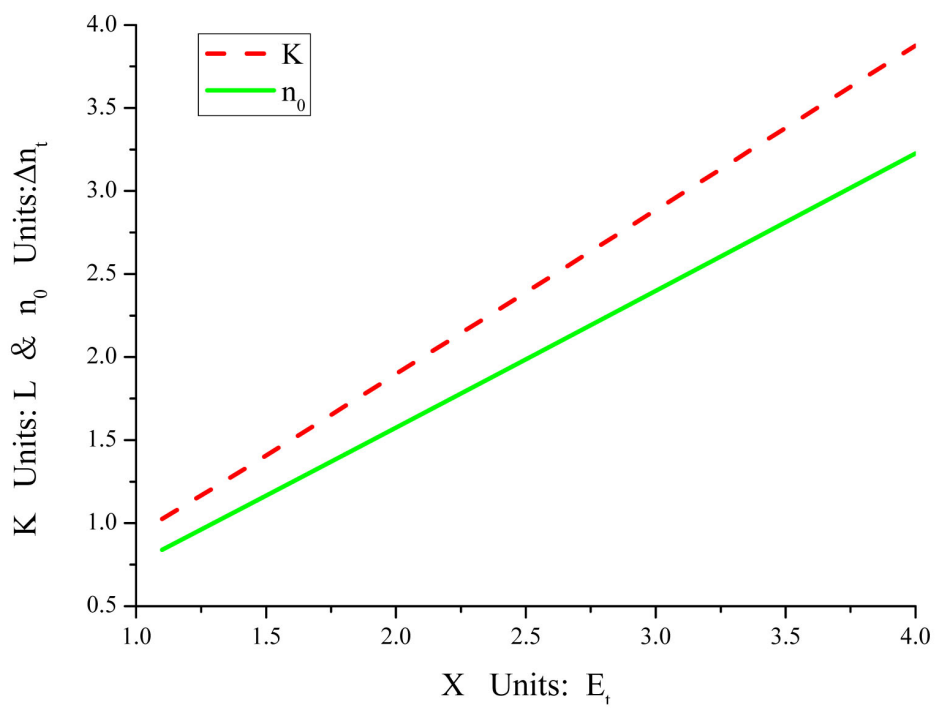

Figure 3. The relationamong the injected energy, actual gain of central mode and loss at the stage of the competition is shown. 
For normal Q-switched laser, the loss at the Q-switched decides the gain of the laser and the curves of them are coincident. It is the main difference between normal Q-switched and pre-lase Q-switched, and the phenomenon is inevitable. The reason is given: The loss at the stage of the competition in pre-lase Q-switched have the same effect as the loss at the stage of Q-switch in normal Q-switched, but an extremely short time costs in the pre-lase Q-switched to keep the mode competition. In extreme time, the part of the inversion population is consumed by the loss of round-trip and the other modes, and the curve gets not coincident. Another thing to mention is that the number of longitudinal modes will increase and the strength of the initial central mode in the seed signal will decrease when the injected energy increases. Therefore, the central mode could not be amplified at the same rate as the injected energy increases after the oscillation and amplification. That is why the growth rate of the actual gain of central mode is inferior to the loss on the competition stage.

\subsection{The Model of the Mode Competition}

Next, the process of competition between the central mode and adjacent mode should be discussed and the fundamental theory shows the power ratio of different mode:

$$
S=\frac{P_{0}}{P_{1}}=\left[\frac{e^{2 \sigma_{0} n_{k} l}}{e^{2 \sigma_{1} n_{k} l}}\right]^{q}\left[\frac{L_{0}}{L_{1}}\right]^{q}
$$

In the Equation (12), $L_{0}, L_{1}$ are the loss of central mode and adjacent mode respectively, and $S \geq 10$. It can be seen that the SLM can be obtained by controlling the loss or extending the time cost during the competition. If the loss device is introduced, the output energy should be influenced and fall off. So it is better to obtain SLM by modulating the time and avoid adding any loss devices in the low energy laser. As we knew, the stimulated emission cross-section $\sigma_{0}$, $\sigma_{1}$ are proportional to the gain $g_{0}, g_{1}$, then the Equation (13)can be obtained by combining Equation (8) and Equation (12):

$$
S=\exp \left[\left(1+\frac{X-1}{4}\left(\frac{\Delta v_{D}}{\Delta v}\right)^{2}\right)-l / l_{0} c \sigma_{n} K \Delta n t\right]
$$

Substitute the optimized loss of competition $K$ and the injected energy $X$ (The optimized parameters are shown in Figure 3) into the Equation (13), then the injected energy and the power ratio of central mode and adjacent mode can be shown in Figure 4.

Figure 4 shows the relation between the power ratio of different modes and injected energy. In fact, the power ratio of different mode decides the stability of SLM. It can be found that the SLM could be realized when the injected energy reaches to 3.25 times of threshold energy. If the injected energy is exceeded, the power ratio of central mode and adjacent mode will be less than ten and the SLM modulation will fail.

At last, the output characters can be acquired by J.JOHN's theory [20]: 


$$
\begin{gathered}
T=1-\exp \left[-L \cdot\left(\frac{z-1-\ln z}{\ln z}\right)\right] \\
E=\frac{A h v L}{2 \sigma \gamma}(z-1-\ln z) \\
\tau_{p}=\frac{2 l}{c L}\left[\frac{\ln z}{z\left(1-\frac{z-1}{z \ln z}\left(1-\ln \frac{z-1}{z \ln z}\right)\right)}\right]
\end{gathered}
$$

where the value of $Z$ is multiple of threshold which is equal to $n_{0}, A=\pi r^{2}$ is the laser beam cross-section, $r=300 \mu m$ is the radius of cross-section, $T$ is the optimum transmittance, $E$ is the single pulse energy, $\tau_{p}$ is the pulse width. Then the output characters of normal Q-switched and pre-lase Q-switched can be respectively computed and shown in Table 1 when the ultimate energy $E_{\text {pump }}=X \cdot E_{t}=585 \mu \mathrm{J}$ is injected.

From Table 1, it can be seen that the output characters decline when the pre-lase technology is applied. The reason for the phenomenon can be given as follows: In the normal Q-switched, all of the population inversion is used to produce the pulsed laser. But the whole population inversion is distributed in

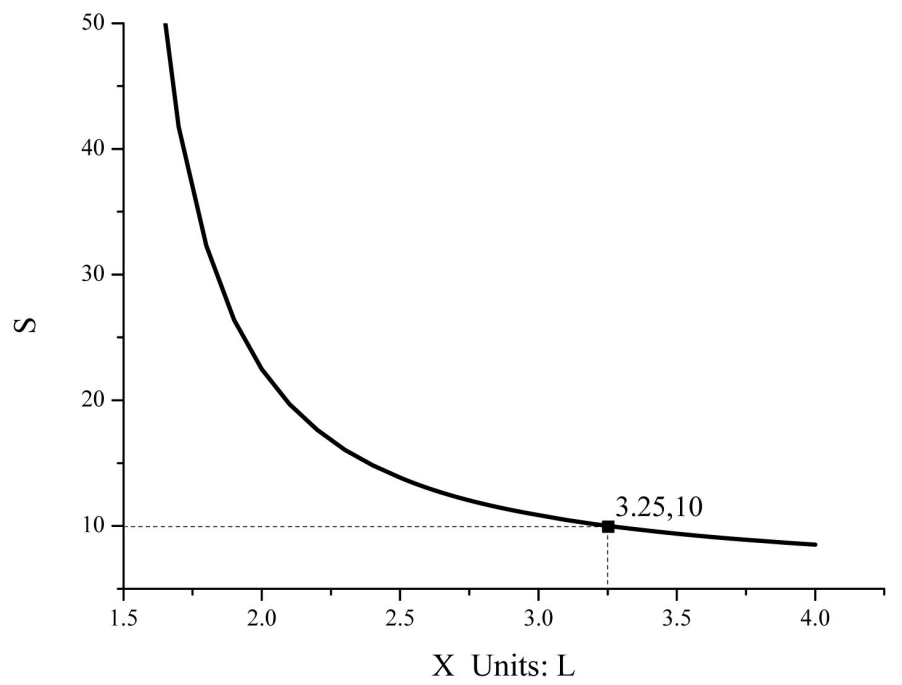

Figure 4. The relation between the injected energy and power ratio of the different modes is shown.

Table 1. The comparison between normal Q-switched and pre-lase Q-switched when the ultimate energy is injected.

\begin{tabular}{ccccccc}
\hline $\begin{array}{c}\text { Different } \\
\text { Q-switched }\end{array}$ & $K(L)$ & $t_{1}(\mu s)$ & $Z\left(\Delta n_{t}\right)$ & $T(\%)$ & $E(\mu)$ & $\tau_{p} \quad(n s)$ \\
\hline $\begin{array}{c}\text { Normal } \\
\text { Q-switched }\end{array}$ & - & - & 3.25 & 3.57 & 141.64 & 10.87 \\
$\begin{array}{c}\text { Pre-lase } \\
\text { Q-switched }\end{array}$ & 3.14 & 35.7 & 2.6 & 2.66 & 85.209 & 15.189 \\
\hline
\end{tabular}


several ways, and the consumption of population inversion is very considerable in the pre-lase. So, it is necessary to optimize the population inversion distribution and the optimized output characters are more close to the normal Q-switched.

\section{Discussion and Conclusion}

In this paper, pre-lase technology is applied to low energy Pr:YLF laser to get efficient SLM laser. It is proved that the output characters are greatly influenced by the injected energy and pre-lase is an efficient method for low energy laser to obtain SLM. After our calculation and analysis, the single pulse energy is, optimum transmittance is and pulse width is when the injected energy reaches the ultimate value and the SLM is just achieved. Finally, the most noteworthy is that the optimization method can be used in the lowest energy solid laser to obtain efficient SLM through the simple control of the loss and avoid introducing any extra loss devices.

\section{Acknowledgements}

This work is supported by The National Natural Fund Project of China (11974060).

\section{Conflicts of Interest}

The authors declare no conflicts of interest regarding the publication of this paper.

\section{References}

[1] Wu, C.T., Ju, Y.L., Zhou, R.L., Duan, X.M. and Wang, Y.Z. (2011) Achieving Single-Longitudinal-Mode Output about Tm:YAG Laser at Room Temperature. Laser Physics, 21, 372-375. https://doi.org/10.1134/S1054660X11030212

[2] Sridhar, G., Rawat, V.S., Kawade, N., Singh, S. and Gantayet, L.M. (2010) Physics and Technology of Tunable Pulsed Single Longitudinal Mode Dye Laser. Pramana, 75, 807-816. https://doi.org/10.1007/s12043-010-0163-Z

[3] Liaw, S.K., Wang, S., Shin, C.S., Chen, N.K., Hsu, K.C. and Manshina, A. (2010) Single-Longitudinal-Mode Linear-Cavity Fiber Laser Using Multiple Subring-Cavities. Laser Physics, 20, 1608-1611. https://doi.org/10.1134/S1054660X10130128

[4] Ahmad, H., Azhari, N.S., Zulkifli, M.Z., Muhammad, F.D. and Harun, S.W. (2014) S-Band SLM Distributed Bragg Reflector Fiber Laser. Laser Physics, 24, 24. https://doi.org/10.1088/1054-660X/24/6/065109

[5] Davey, R.P., Fleming, R.P.E., Smith, K., Kashyap, R. and Armitage, J.R. (1991) Mode-Locked Erbium Fibre Laser with Wavelength Selection by Means of Fibre Bragg Grating Reflector. Electronics Letters, 27, 2087-2088. https://doi.org/10.1049/el:19911292

[6] Baxter, G.W., Schlup, P. and Mckinnie, I.T. (2000) Efficient, Single Frequency, High Repetition Rate, PPLN OPO Pumped by a Prelase Q-Switched Diode-Pumped Nd:YAG Laser. Applied Physics B, 70, 301-304.

https://doi.org/10.1007/s003400050049 
[7] Owyoung, A., Hadley, G.R., Esherick, P., Schmitt, R.L. and Rahn, L.A. (1985) Gain Switching of a Monolithic Single-Frequency Laser-Diode-Excited Nd:YAG Laser. Opt Lett (United States), 10, 484-486. https://doi.org/10.1364/OL.10.000484

[8] Crawford, T., Lowrie, C. and Hompson, J.R. (1996) Prelase Stabilization of the Polarization State and Frequency of a Q-Switched, Diode-Pumped, Nd:YAG Laser. Applied Optics, 35, 5861-5869. https://doi.org/10.1364/AO.35.005861

[9] Resan, B. and Coadou, E. (2011) Ultrashort Seed-Pulse Generating Laser with Integral Pulse Shaping. US 7894493 B2.

[10] Barnes, N.P. and Barnes, J.C. (1993) Injection Seeding I: Theory. IEEE Journal of Quantum Electronics, 29, 2670-2683. https://doi.org/10.1109/3.250390

[11] Yu, J.R., Singh, U.N. and Barnes, P. (1988) 125-mJ Diode-Pumped Injection Seeded Ho:Tm:YLF Laser. Optics Letters, 23, 780-782. https://doi.org/10.1364/OL.23.000780

[12] Hanna, D.C., Luther-Davies, B., Rutt, H.N. and Smith, R.C. (1971) A Two-Step Q-Switching Technique for Producing High Power in a Single Longitudinal Mode. Opto-Electronics, 3, 163-169. https://doi.org/10.1007/BF01419332

[13] Xu, B., Liu, Z., Xu, H., Cai, Z., Zeng, C. and Huang, S. (2013) Highly Efficient InGaN-LD-Pumped Bulk Pr:YLF Orange Laser at $607 \mathrm{~nm}$. Optics Communications, 305, 96-99. https://doi.org/10.1016/j.optcom.2013.05.002

[14] Li, X.D., Yu, X., Yan, R.P., Fan, R.W. and Chen, D.Y. (2011) Optical and Laser Properties of $\operatorname{Pr}^{3+}$ :YLF Crystal. Laser Physics Letters, 8, 791-794.

https://doi.org/10.1002/lapl.201110069

[15] Jelínková, H. and Fibrich, M. (2009) Electro-Optically Q-Switched Pr:YAP Laser Generating at $747 \mathrm{~nm}$. Laser Physics Letters, 6, 517-520. https://doi.org/10.1002/lapl.200910028

[16] Fu, X.H., Li, Y.L. and Jiang, H.L. (2011) Diode-Pumped $\mathrm{Pr}^{3+}: \mathrm{YAlO}_{3} / \mathrm{LBO}$ Violet Laser at $374 \mathrm{~nm}$. Laser Physics, 21, 864-866. https://doi.org/10.1134/S1054660X11090088

[17] Svelto, O. (2010) Principles of Lasers. Springer. https://doi.org/10.1007/978-1-4419-1302-9

[18] Dai, T.Y., Wu, J. and Zhang, Z.G. (2015) Diode-End-Pumped Single-LongitudinalMode Er:LuAG Laser with Intracavity Etalons at 1.6 $\mu \mathrm{m}$. Applied Optics, 54, 9500. https://doi.org/10.1364/AO.54.009500

[19] Sooy, W.R. (1965) The Natural Selection of Modes in a Passive Q-Switched Laser. Applied Physics Letters, 7, 36-37. https://doi.org/10.1063/1.1754286

[20] Degnan, J.J. (1989) Theory of the Optimally Coupled Q-Switched Laser. IEEE Journal of Quantum Electronics, 25, 214-220. https://doi.org/10.1109/3.16265 